Note: This is a draft of a paper being submitted for publication. Contents of this paper should not be quoted nor referred to without permission of the author(s).

To be submitted to Fall 2000 Materials Research Society Meeting.

\title{
Effects of ArF Excimer Irradiation on Multi-Energy Ge and Se Ion Implanted Silica
}

\author{
R. H. Magruder III \\ Belmont University, Nashville, TN
}

R. A. Weller, R. A. Weeks, and J. Wehrmeyer

Vanderbilt University, Nashville, TN

R. A. Zuhr and D. K. Hensley

Oak Ridge National Laboratory, Oak Ridge, TN

\begin{abstract}
"The subunitted manuscript has been authored by a contractor of the U.S. Government under contract No. DE-AC05-00OR22725. Accordingly, the U.S. Government retains a nonexclusive, royalty-free license to publish or reproduce the published form of this contribution, or allow others to do so, for U.S. Government purposes."
\end{abstract}

\author{
Prepared by the \\ Oak Ridge National Laboratory \\ Oak Ridge, Tennessee 37831 \\ managed by \\ UT-Battelle, LLC \\ for the \\ U.S. DEPARTMENT OF ENERGY \\ under contract DE-AC05-00OR22725
}

November 2000 


\title{
Effects of ArF Excimer Irradiation on Multi-Energy Ge and Se Ion Implanted Silica
}

\begin{abstract}
R. H. Magruder, III, ${ }^{1}$ R. A. Weller ${ }^{2}$, R. A. Weeks ${ }^{3}$, J. Wehrmeyer ${ }^{3}$, R. A. Zuhr ${ }^{4}$ and D. K. Hensley, ${ }^{4}$ 1Dept. of Physics, Belmont University, Nashville, TN 37212, ${ }^{2}$ Dept. of Electrical Engineering and Computer Science, Vanderbilt University, Nashville TN 37235, ${ }^{3}$ Dept. of Mechanical Engineering, Vanderbilt University, Nashville TN 37235, 4 Solid State Division, Oak Ridge National Laboratory, Oak Ridge, TN 37831
\end{abstract}

\begin{abstract}
Silica samples were implanted at multiple energies with Se and Ge ions producing implanted layers $\sim 2.4 \mu \mathrm{m}$ in depth starting at $\sim 0.5 \mu \mathrm{m}$ below the surface. In each case, the concentration of implanted species was $\sim 0.05$ atomic $\%$. The optical absorption of the samples was measured from 2.7 to $6.5 \mathrm{eV}$. In all spectra, local maxima at $5.0 \mathrm{eV}$ with shoulders at $5.9 \mathrm{eV}$ were observed. The spectra have been assumed to be describable as a superposition of Gaussian absorption bands with mean energies taken from the literature of 4.8,5.01, 5.17, 5.88, and 7.15 eV. The relative strengths of each of these bands have been obtained by linear regression and the variability of these strengths measured as a function of excimer laser irradiation. These fits show that additional bands at 3.7 and $6.4 \mathrm{eV}$, whose parameters have been obtained by nonlinear methods, are required to fit the data for the Se implanted samples, while bands at 5.54 and $6.4 \mathrm{eV}$ are needed to fit the absorption of the Ge implanted samples.
\end{abstract}

\section{INTRODUCTION}

Devices using photoinduced fiber Bragg gratings have been demonstrated or postulated for new ways of optical switching, and for signal processing in fiber and waveguide systems[1]. Implanting silica with $\mathrm{Si}, \mathrm{Ge}, \mathrm{O}$, or $\mathrm{H}$ ions has been shown to produce photosensitive defects in silica $[2,3,4,5]$. Changes in the index of refraction, $\Delta \mathrm{n}$, of $\sim 0.1 \%$, with excimer radiation have been reported [3,4] and attributed to the photobleaching of oxygen related defect centers. The optically active defect sites most often correlated with the index changes have absorption maxima in the 4.8 to $8.0 \mathrm{eV}$ region $[1,2,3,4]$.

The purpose of this work is to measure the photobleaching of optical absorption bands by ArF excimer laser irradiation. The absorption bands have been produced by multi-energy Se and Ge implantations in (Type III) silica. We have used multiple energy implantations to create a larger chemical interaction region with an approximately constant ion species distribution throughout. We have chosen $\mathrm{Ge}$ and Se because, while the mass and nuclear charge of $\mathrm{Ge}$ and $\mathrm{Se}$ are similar, resulting in similar radiation damage during the implantation, the chemistry of these two ions differs markedly. Ge substitutes for $\mathrm{Si}$ in the network, while Se can be expected to replace $O$. Ge ions have been shown to exist in both 2 fold and 4 fold coordination [1]. With the implantation of Ge we can expect the implanted region to be metal rich, in which case there may also be $\mathrm{Ge}^{2+}$ present. While Se can chemically substitute for oxygen in coordination number, it does have some significant differences in bonding with $\mathrm{Si}[6,7]$. In the case of oxygen, we expect the normal tetrahedrally coordinated covalent solid, whereas $\mathrm{SiSe}_{2}$ is thought to consist of edge sharing tetrahedral chains. These chains are then cross linked by corner sharing tetrahedra $[6,7]$. 
We fit the observed optical absorption spectra of the Ge- and Se-implanted samples to a superposition of absorption bands reported in the literature $[8,9,10]$. The effects of the ion species and ArF irradiation on the amplitudes of these intrinsic bands are examined.

\section{EXPERIMENTAL}

Samples were prepared by implanting Se or Ge ions using multiple energies (ME) in sequence ranging from $4 \mathrm{MeV}$ to $0.7 \mathrm{MeV}$ with areal densities as shown in Table $\mathrm{I}$.

\section{Table I. Doses as Functions of Energies}

\begin{tabular}{|l|l|l|l|l|}
\hline $\begin{array}{l}\text { Energy } \\
(\mathrm{MeV})\end{array}$ & $\begin{array}{l}\text { Se ME sample } \\
\text { Areal density } \\
\times 10^{15} \text { ions } / \mathrm{cm}^{2}\end{array}$ & $\begin{array}{l}\text { Se ME sample } \\
\text { Areal density } \\
\mathrm{x} 10^{15} \text { ions } / \mathrm{cm}^{2}\end{array}$ & $\begin{array}{l}\text { Ge ME sample } \\
\text { Areal Density } \\
\times 10^{15} \text { ions } / \mathrm{cm}^{2}\end{array}$ & $\begin{array}{l}\text { Ge ME sample } \\
\text { Areal density } \\
\times 10^{15} \text { ions } / \mathrm{cm}^{2}\end{array}$ \\
\hline $4 \mathrm{MeV}$ & 5.0 & 2.5 & 5.0 & 2.5 \\
\hline $2.7 \mathrm{MeV}$ & 1.6 & 0.8 & 1.6 & 0.8 \\
\hline $1.95 \mathrm{MeV}$ & 1.4 & 0.7 & 1.4 & 0.7 \\
\hline $1.4 \mathrm{MeV}$ & 0.85 & 0.43 & 0.85 & 0.43 \\
\hline $1 \mathrm{MeV}$ & 0.61 & 0.31 & 0.61 & 0.31 \\
\hline $0.7 \mathrm{MeV}$ & 0.5 & 0.25 & 0.5 & 0.25 \\
\hline
\end{tabular}

The fluences at each energy were calculated to maintain an approximately constant concentration of the implant species over the complete range of the implanted ions. The implanted substrates were Type III silica (Corning 7940). All implantations were carried out at a current density of $\sim 500 \mathrm{nA} / \mathrm{cm}^{2}$ and a substrate temperature of $273 \mathrm{~K}$.

The optical absorption was measured at wavelength intervals of $1 \mathrm{~nm}$ from $2.7 \mathrm{eV}$ to $6.5 \mathrm{eV}$ using a dual beam (Cary 5) spectrometer with an unimplanted sample in the reference beam. Hence, all absorption measurements represent the differences between implanted and unimplanted samples and are reported in units of optical density.

Samples were photobleached using a total of 3,11, and 31 pulses of $6.4 \mathrm{eV} \mathrm{ArF} \mathrm{excimer} \mathrm{laser}$ radiation at an energy density of $44 \mathrm{~mJ} / \mathrm{cm}^{2}$ per pulse. After each cycle of photobleaching, the optical absorption spectrum was measured.

\section{RESULTS}

From calculations using the computer program "Profile" [11], the implanted layer is inferred to have an approximately constant concentration of Se that starts $\sim 0.5 \mu \mathrm{m}$ below the surface and extends for $\sim 2.4 \mu \mathrm{m}$. Similarly, the implanted Ge layer, also of constant concentration, starts $0.8 \mu \mathrm{m}$ below the surface and extends for $\sim 2.6 \mu \mathrm{m}$. The concentrations of Se in the samples are $\sim 0.048$ atomic $\%$ and 0.024 atomic $\%$. The concentrations of Ge are similarly $\sim 0.042$ atomic $\%$ and $\sim 0.021$ atomic $\%$.

Figure 1 shows the optical density for the sample implanted with Se to a concentration of 0.048 atomic \% before and after each $\mathrm{ArF}$ excimer exposure. The absorption spectrum has a resolved peak at $\sim 5 \mathrm{eV}$ and a shoulder at $\sim 5.9 \mathrm{eV}$ with rapidly increasing absorption for photon energies greater than $6 \mathrm{eV}$. The optical absorption for the sample after 3,11, and 31 pulses of 
ArF irradiation is shown. Even the smallest doses of $\mathrm{ArF}$ irradiation were observed to change the optical absorption. With increasing ArF irradiation, the absorption decreases for photon energies $>4.5 \mathrm{eV}$. However, the ratio of optical density at $5.9 \mathrm{eV}$ to that at $5 \mathrm{eV}$ remains constant.

Bleaching in the sample with the smaller Se concentration, 0.024 atomic $\%$, is similar to that in the sample with the larger Se concentration.

Figure 2 shows the optical density of the sample implanted with Ge to a concentration of 0.042 atomic \% before and after each $\mathrm{ArF}$ excimer exposure. After the first ArF exposure, there is a decrease of the optical density for photon energies greater than $4.5 \mathrm{eV}$, with the largest change at photon energies $>5.5 \mathrm{eV}$.

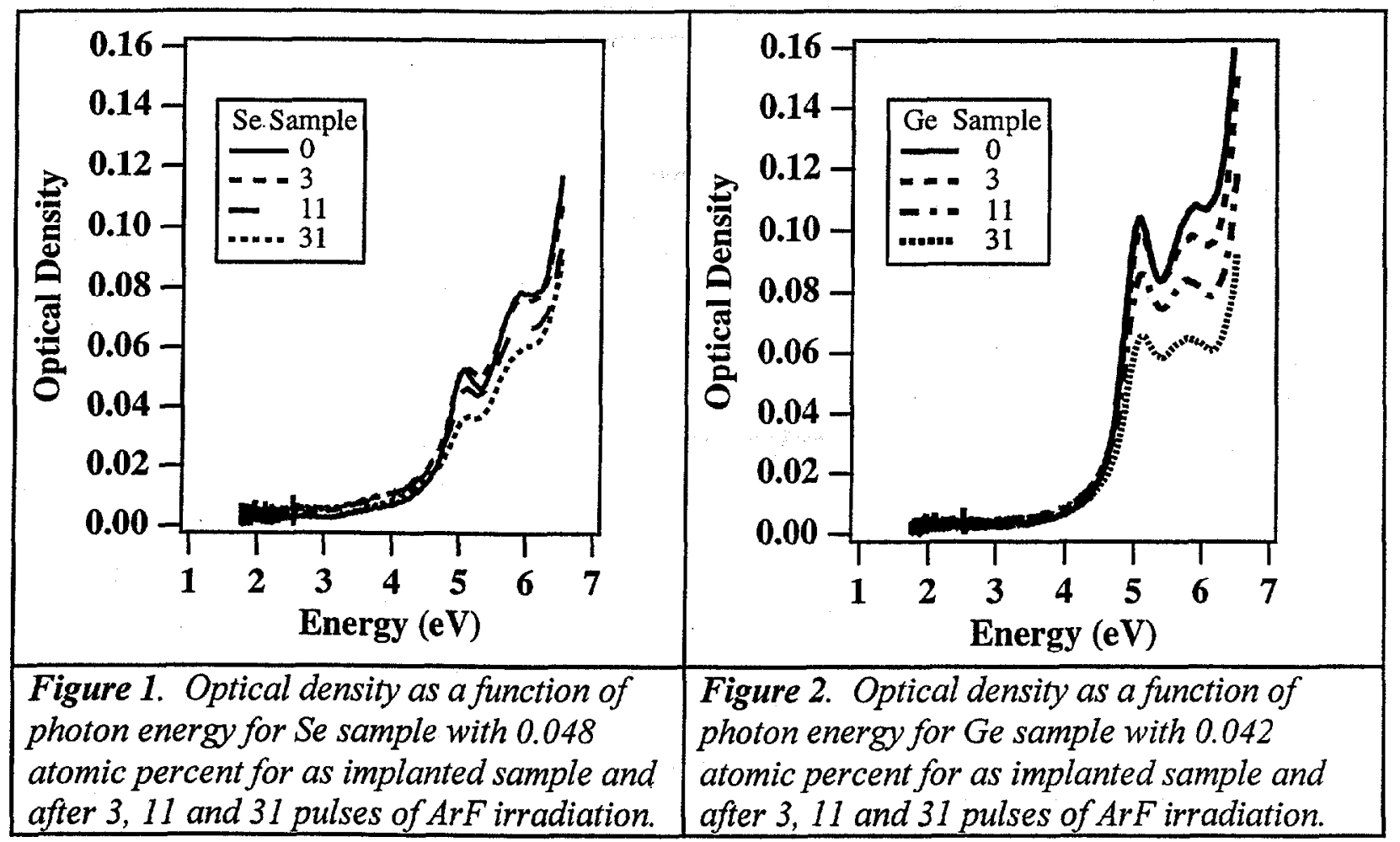

Subsequent ArF irradiation reduces the optical density for photon energies greater than $\sim 4.5 \mathrm{eV}$. After 31 pulses of ArF radiation the relative magnitude of the optical density at $5 \mathrm{eV}$ and $5.9 \mathrm{eV}$ remains similar to the as-implanted state, though both are reduced in magnitude. Bleaching in the sample with the smaller Ge concentration, 0.021 atomic \%, is similar to that in the sample with larger Ge concentration.

\section{DISCUSSION}

Near the surface, ion energy loss occurs primarily by inelastic collisions that produce electronic excitations. Near the end of the ion track, elastic collisions between the incident and substrate nuclei dominate, leading to more atomic displacements. We assume that it is in this region, where most of the implanted ions come to rest, that most of the chemical interactions between implanted ions and the host matrix occur. Chemical interactions with the implanted ions can result in additional defect structures. One of the primary goals of using multiple implant energies was to create a larger chemical reaction region in which the implanted concentration is 
approximately constant. This region may permit us to resolve the effects of implanted ion species on the optical absorption. In the Ge implanted samples, the interaction layer is $\sim 2.6 \mu \mathrm{m}$ thick, while in the Se implanted samples it is about $\sim 2.5 \mu \mathrm{m}$ thick.

The displacement damage caused by the $\mathrm{Ge}$ and Se ions is similar, since the ion mass, nuclear charge, implant energies, and areal densities were comparable. Likewise, because the implanted regions are of similar thickness and the concentrations of implanted ions are similar ( 0.048 atomic $\%$ for the Se and 0.042 atomic $\%$ for the Ge), optical effects due to variations in concentration or size of the implantation region are minimal. If the absorption producing mechanism were dominated by inelastic collision damage, it is reasonable to expect the magnitude of the optical absorption of the Ge and Se samples to be similar. From Figs. 1 and 2 the optical density is ion species dependent for energies greater than $\sim 3.0 \mathrm{eV}$. We conclude from these observations that the presence of the Se and $\mathrm{Ge}$ ions affects defect production and bleaching differently.

In previous work it was assumed that the optical absorption produced by ion implantation was due to intrinsic defect states reported in the literature [12,13]. At least eight overlapping optical bands are reported in the literature between 2 and $8.0 \mathrm{eV}[14,15,16,17,18]$. This overlap makes unambiguous identification of bands and assignment of band strengths difficult without a careful analysis of the spectra.

While there have been several models proposed for the absorption at $4.8 \mathrm{eV}$, the $\mathrm{D}_{\mathrm{o}}$ center, a correlation with the peroxy radical electron paramagnetic resonance, has been reported, as well as a correlation with an $\mathrm{O}_{3}$ molecule $[8,14]$. The absorption band at $5.01 \mathrm{eV}$, the $\mathrm{E}^{\prime \prime}\left(\mathrm{B}_{2}\right)$ center, is attributed to a neutral oxygen vacancy [19]. The absorption band at $5.17 \mathrm{eV}$, the $\mathrm{B}_{2 \beta}$ center, has been attributed to two coordinated $\mathrm{Ge}$ or $\mathrm{Si}$ atoms (an oxygen divacancy) [14,20]. These three defects, the $E^{\prime \prime}, B_{2} \beta$ and $D_{0}$, have absorption between 4.8 and $5.2 \mathrm{eV}$ in silica and germanium doped silica. The absorption band at $5.88 \mathrm{eV}$, the $\mathrm{E}^{\prime}$ center, is attributed to a charged oxygen vacancy $[14,15]$. Correlated with the absorption band of the $E^{\prime \prime}$ at $5.01 \mathrm{eV}$ is an absorption at $7.6 \mathrm{eV}$, which Imai et al. [21] have suggested is related to the same defect structure, a neutral oxygen vacancy. The $5.01 \mathrm{eV}$ absorption is assumed to be an unrelaxed oxygen vacancy and the $7.6 \mathrm{eV}$ absorption is assumed to be the relaxed oxygen vacancy. There is also a band, labeled the $\mathrm{D}$ band by Antonini et al., that is reported at $7.15 \mathrm{eV}$ [18]. This band is also assumed to be an oxygen related defect center [17].

To study the effects of ion species on these defect bands, we fit the observed spectra in Figs. 1 and 2 to a superposition of Gaussian basis states with positions and widths taken from the literature. We have chosen the minimum number of bands required to fit the data to within \pm 2 $\%$. For linear fits, the amplitude of each band is a fit parameter along with a single constant background $[12,13,16]$. We used the following bands, the $4.8 \mathrm{eV}$ with FWHM of $1.1 \mathrm{eV}$, the $5.01 \mathrm{eV}$ with FWHM of $0.35 \mathrm{eV}$, the $5.17 \mathrm{eV}$ with FWHM of $0.5 \mathrm{eV}$, the $5.88 \mathrm{eV}$ with FWHM of $0.9 \mathrm{eV}$, and the $7.15 \mathrm{eV}$ with a FWHM of $0.82 \mathrm{eV}$. We also used a band at $6.4 \mathrm{eV}$ with a FWHM of $0.4 \mathrm{eV}$ that we have previously described $[13,16]$. This band was required to achieve fitting to $\pm 2 \%$ for the energies $>5.8 \mathrm{eV}$. While the structure of the defect leading to a $6.4 \mathrm{eV}$ band has not been described in the literature, in the discussion below we assume that the $6.4 \mathrm{eV}$ band is the result of a defect produced by ion implantation. Even with the presence of the 7.15 $\mathrm{eV}$ band, the band at $6.4 \mathrm{eV}$ was required for fitting for energies greater than $5.9 \mathrm{eV}$. While there are bands at energies $>6.5 \mathrm{eV}$, our data are limited to energies $\leq 6.5 \mathrm{eV}$, and we have used the $7.15 \mathrm{eV}$ band to represent bands at energies $>6.5 \mathrm{eV}$ in these fits. In the case of the $\mathrm{Ge}$ implanted samples, these fits did not fit the data to within $+2 \%$ in the 5.4 to $6.0 \mathrm{eV}$ region of the 
spectra. In the case of the Se implanted samples these fits did not fit the data to within $\pm 2 \%$ in the 3.2 to $4.5 \mathrm{eV}$ region of the spectra.

A non-linear regression was used to determine the position, width, and amplitude of the single additional band used to produce the minimum chi-squared. In the case of the Ge implanted samples the result of this approach was the consistent inference of an additional band at $5.54 \mathrm{eV}$ with a FWHM equal to $0.32 \mathrm{eV}$. The additional band produced a fit that was within $\pm 2 \%$ over the entire region from 2.7 to $6.5 \mathrm{eV}$. In the same manner, this procedure was used to analyze the Ge samples exposed to laser irradiation. In all cases the adjustable band was found to be 5.54 $\pm 0.05 \mathrm{eV}$, with a consistent FWHM of $0.32 \pm 0.05 \mathrm{eV}$.

This procedure was then used to fit the spectra of the Se implanted samples. One result was the consistent inference of an additional band at $3.7 \pm 0.1 \mathrm{eV}$ with a FWHM equal to $1.1 \pm 0.1 \mathrm{eV}$, for a fit within $\pm 2 \%$ over the region from 3.0 to $6.5 \mathrm{eV}$. The band at $5.54 \mathrm{eV}$ was not required in the Se implanted samples. The same procedure was used to analyze the Se samples exposed to laser irradiation. In all cases the adjustable band was found to be $3.7 \mathrm{eV}$ with a consistent FWHM of $1.1 \mathrm{eV}$. We have previously reported this band for single energy implanted Se implanted glasses [12].

The amplitudes of the various Gaussian bands obtained from the nonlinear fits to data from the Ge implanted samples are plotted in Figure 3. Figure 4 contains similar plots for the Se implanted samples. These relative magnitudes may be used to infer the concentrations of defects through Samakula's equation, provided the oscillator strengths of the absorptions are known [8].

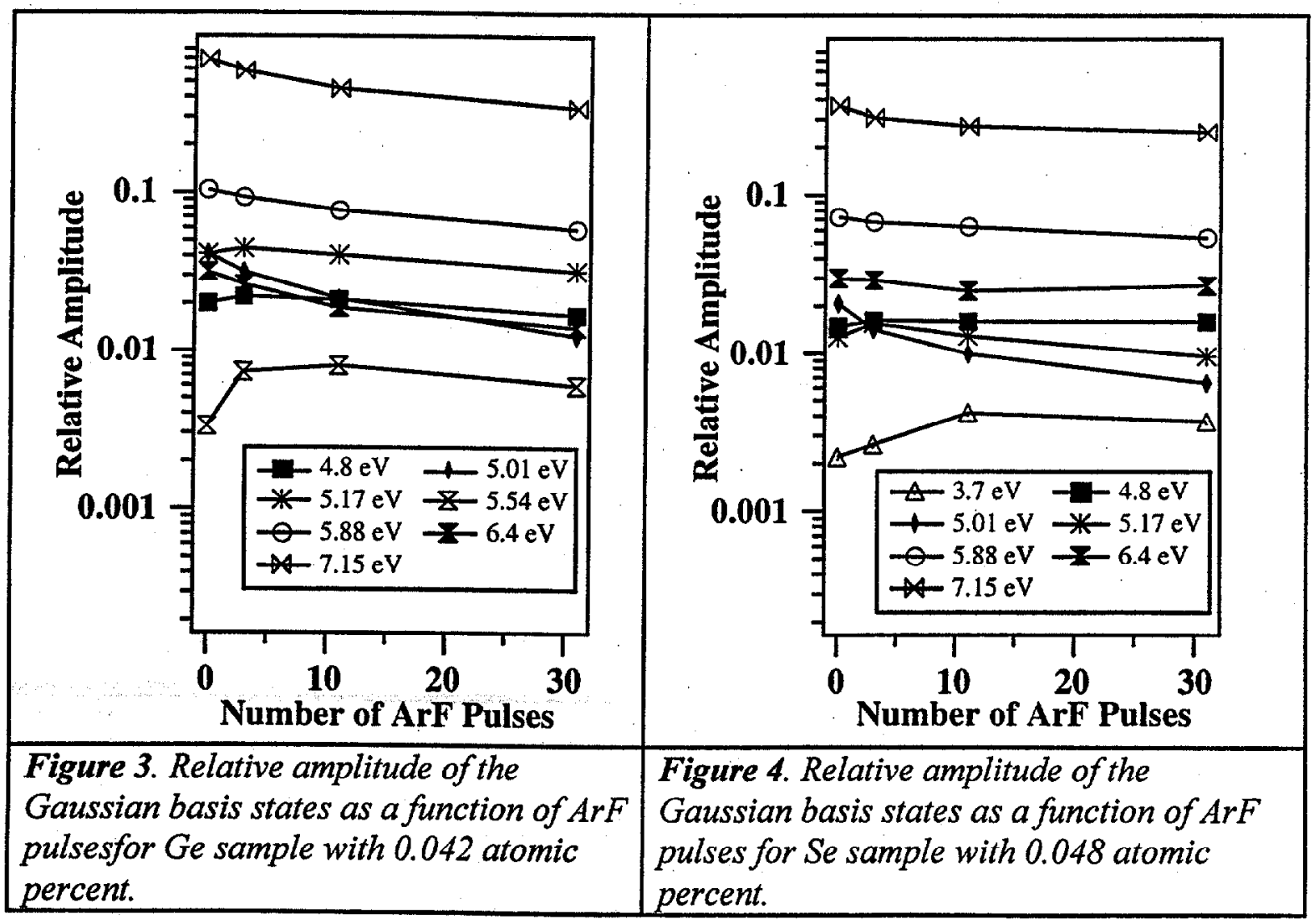


We will use these magnitudes without modification as indirect measures of the concentrations of defects present in each band. Calculation of concentrations of the various defects is not done because the oscillator strength of only one band, the E' center, is known [14].

In the as-implanted samples, all the absorption bands are weaker in Se implanted samples than in Ge implanted samples. All the bands, except the 3.7 and $6.4 \mathrm{eV}$ bands in the Se sample and the $5.54 \mathrm{eV}$ band in the Ge sample, decrease with exposure to ArF irradiation.

One of the effects of Ge implantation is to make the implanted volume oxygen deficient. We expect that two consequences of this change are to increase the relative numbers of oxygen deficient centers, such as $E^{\prime}$, neutral oxygen vacancies, and oxygen divacancies in their various charge states, and to increase the fraction of defects such as $\mathrm{Si}-\mathrm{Si}, \mathrm{Si}-\mathrm{Ge}$, and $\mathrm{Ge}-\mathrm{Ge}$ dimers, and fewer trimers of these two ions. The 5.01, 5.17, 5.88 and $7.15 \mathrm{eV}$ bands are suggested to be oxygen deficient sites $[4,8,9,14]$. In the case of the Se implants we expect Se to replace the oxygen. Based on the results of Figures 3 and 4 we conclude that Se does replace $O$, since the bands associated with oxygen deficiency are weaker in Se implanted samples than in Ge implanted samples. Moreover, the $4.8 \mathrm{eV}$ band has been associated with the peroxy radical and has also been suggested to be associated with a $\mathrm{Se}_{2}{ }^{-}$structure in Se implanted silica $[12,14,15]$. Since the $5.54 \mathrm{eV}$ band is not observed in the Se implanted samples, we conclude that it is related to the presence of $\mathrm{Ge}$. This is consistent with samples comparably implanted with $\mathrm{Si}, \mathrm{B}, \mathrm{Ti}$, and $\mathrm{Se}$, where the $5.54 \mathrm{eV}$ band was not required to achieve satisfactory fits to the data $[12,13,16]$. The $3.7 \mathrm{eV}$ band is required for the $\pm 2 \%$ fitting only in the Se implanted samples. This band has been previously attributed to a $\mathrm{Si} \mathrm{Se}_{2}{ }^{-}$structure in silica [12]. This is consistent with our results. The magnitude of the $6.4 \mathrm{eV}$ band is the same for both the Se and $\mathrm{Ge}$ implanted samples. Based on the consistency of location and width of the $6.4 \mathrm{eV}$ band, we postulate that it is a real feature of these absorption spectra. It is, of course, possible that it is an artifact arising from the cumulative effects of one or more bands in the vacuum UV region outside of the range of our data. However, this band has been required to fit spectra of silica implanted with Ti, B and Si to similar doses and with similar energies $[12,13,16]$. We conclude, therefore, that it is not specific to the presence of $\mathrm{Ge}$ or $\mathrm{Se}$ and is an intrinsic defect, possibly associated with radiation damage. While there are some differences in the bleaching of the various bands, the major effect of the implanted ion species is the production of defects intrinsic to silica. For the ion concentrations used in this work, we conclude that the defect populations are affected by the chemistry of the implanted ion species and their interaction with the silica.

\section{CONCLUSION}

The bands at 4.8,5.01 5.17, 5.88, 6.4 and $7.15 \mathrm{eV}$ produced by implanting $\mathrm{B}, \mathrm{Si}$ and $\mathrm{Ti}$ are reproduced in these samples implanted with $\mathrm{Ge}$ and Se. The implanted Se acts to reduce the amplitude of the bands associated with oxygen deficiencies compared to the $\mathrm{Ge}$ implanted samples. In addition the band at $3.7 \mathrm{eV}$ produced by Se implantation is due to a chemical state of Se. In the Ge case we conclude that the band at $5.54 \mathrm{eV}$ is due to a chemical state of Ge. Bleaching in the $\mathrm{Se}$ and $\mathrm{Ge}$ samples affects bands to approximately the same degree.

\section{ACKNOWLEDGMENT}

The authors acknowledge the support of Oak Ridge National Laboratory, managed by UTBattelle, LLC, for the U.S. Department of Energy under contract number DE-AC05-00OR22725. 